\title{
Fuzzy Temporal Sequence Processing By Fuzzified Recurrent Neural Fuzzy Network
}

\author{
Chia-Feng Juang and Shiuan-Jiun Ku \\ Department of Electrical Engineering, \\ National Chung Hsing University \\ Taichung, 402 Taiwan, R.O.C.
}

\author{
Hao-Jung Huang \\ Department of Electrical Engineering \\ Chung Chou Institute of Technology \\ Yuan-Lin, Chang-Hua, Taiwan, R.O.C.
}

\begin{abstract}
A Fuzzified TSK-type Recurrent Neural Fuzzy Network (FTRNFN) for handling fuzzy temporal information is proposed in this paper. The inputs and outputs of FTRNFN are fuzzy' patterns represented by Gaussian or isosceles triangular membership functions. In structure, FTRNFN is a recurrent fuzzy network constructed from a series of recurrent fuzzy if-then rules with TSK-type consequent parts. The recurrent property of FTRNFN enables it to deal with fuzzy patterns with temporal context. There are no rules in FTRNFN initially; they are constructed on-line by concurrent structure and parameter learning. The ability' of TRFNFN is verified jrom a two-dimensional fuzzy temporal sequence prediction problem.
\end{abstract}

Keywords: fuzzy neural network, TSK-type fuzzy rule, temporal sequence prediction

\section{Introduction}

To process temporal patterns whose output depend not only on the present input but also on those preceding or following it, many recurrent neural/fuzzy networks have been proposed [1]-[4]. In [4], we have proposed a TSK-type recurrent fuzzy network (TRFN). In TRFN, the internal variables, derived from fuzzy firing strengths, are fed back to both network input and output layers in TRFN, and no $a$ priori knowledge of the system order is required which eases the design process. In addition, the performance of TRFN designed by neural networks has been demonstrated to outperform those compared recurrent networks. However, TRFN can process numerical information only. In real world, except numerical information, information may be vague signals from measurement or linguistic information from human experts. To handle this type of information, a network for processing fuzzy input and output is required.

For a neural network to process linguistic information with fuzzy inputs, fuzzy outputs, or fuzzy weights, some approaches have been proposed [5]-[8]. In [5], a fuzzy set was represented by its membership values on the discrete base set. These real membership values were used as input values to neural networks and then the real numbers were propagated through neural networks. In [6], the authors have proposed an architecture of multilayer feedforward

\section{* 0-7803-8566-7/04/\$20.00 두 2004 IEEE.}

neural network that can deal with fuzzy input vectors with fuzzy/crisp outputs. In their method, the inputs and outputs of the neural network are fuzzified using fuzzy numbers represented by $a$-level sets. Besides the aforementioned fuzzified neural networks, in $[7,8]$, neural fuzzy networks for processing fuzzy information are proposed. In these networks, the network structures are feedforward and the consequent parts are fuzzy sets, and the whole network is constructed off-line.

In the aforementioned networks, the structures are all feedforward. To handle temporal fuzzy signals, a fuzzified recurrent network is more suitable than a feedforward network. Based on the good performance of TRFN, in this paper, we will extend it to deal with fuzzy temporal information, and a Fuzzified TSK-type Recurrent Neural Fuzzy Network (FTRNFN) is proposed. In FTRNFN, the consequent part is of TSK type. The fuzzy sets in the precondition part of FTRNFN are Gaussian fuzzy sets, and in the consequent part, the line combination coefficients are of Gaussian fuzzy sets, too. The center and width of every Gaussian fuzzy set in the precondition and consequent parts are all tunable. The input and output fuzzy information may be represented by Gaussian or isosceles triangular fuzzy sets. As to the network learning, in contrast to the off-line learning in [7,8], all recurrent fuzzy rules in FTRNFN are constructed automatically by on-line structure and parameter learning. So the network can be used for normal operation at any time as learning proceeds.

The remaining sections of this paper are organized as follows. Section 2 describes the basic structure and functions of FTRNFN. The online structure/parameter learning algorithms of FTRNFN is presented in Section 3. In Section 4, the FTRNFN is applied to solve twodimensional fuzzy sequence prediction problem. Conclusions are summarized in the last section.

\section{Structure of FTRNFN}

In this section, the structure of FTRNFN (shown in Fig. 1) is introduced. The FTRNFN deals with fuzzy input values and sends out fuzzy output values, where each fuzzy value is represented by a fuzzy set with Gaussian or isosceles triangular membership function. Suppose there are two external fuzzy inputs and two fuzzy outputs in the network, then this six-layered network realizes a recurrent fuzzy reasoning of the following form: 
fuzzy coordinates of the predicted fuzzy point. Aster training, 6 input clusters (rules) are generated. The predicted result is shown in Fig. 3, where a good performance is achieved.

\section{Conclusions}

In this paper, an FTRNFF is proposed for fuzzy temporal sequence processing. FTRNFN is a recurrent network which can learn and memorize the context information of a fuzzy temporal sequence. FTRNFN is characterized with on-line structure and parameter learning. Based on structure learning, flexible partition of the input space is achieved. Thus the total number of rules in FTRNFN can be reduced. Result on the demonstrative example motivates us further applications of FTRNFN on other fuzzy temporal sequence processing problems.

\section{Acknowledgment}

This work was supported by the National Science Council, Taiwan, R.O.C. under Grant NSC-92-2213-E-005001 .

\section{References}

[1] C. L. Giles, G. M. Kuhn, and R. J. Williams, "Dyna:nic recurrent neural networks: theory and applications," IEEE Trans. Neural Networks, vol. 5, pp. 153-156, 1994.

[2] C. H. Lee and C. C. Teng, "Identification and control of dynamic systems using recurrent fuzzy neural networks," IEEE Trans. Fuzzy Systems, vol. 8, no. 4, pp. 349-366, 2000.

[3] C. F. Juang and C. T. Lin, "A recurrent self-organizing neural fuzzy inference network," IEEE Trans. Neural Networks, vol. 10, no. 4, pp. 828-845, 1999.

[4] C. F. Juang, "A TSK-type recurrent fuzzy network for dynamic systems processing by neural network and genetic algorithm," IEEE Trans. Fuzzy Systems, vol. 10, no. 2, pp. 155-170, 2002.

[5] J. M.Keller, R. R. Yager, and H. Tahani, "Neural network implementation of fuzzy logic," Fuzzy Sets and Systems, vol. 45, pp. 1-12, 1992.

[6] H. Ishibuchi, R. Fujioka, and H. Tanaka, "Neural networks that learn from fuzzy if-then rules," IFEE Trans. Fuzzy Systems, vol. 2, pp. 85-97, 1993.

[7] C. T. Lin and Y. C. Lu, "A neural fuzzy system with linguistic teaching signals," IEEE Trans. Fuzzy Systems," vol. 3, no. 2, pp. 169-189, May, 1995.

[8] S. Paul and S. Kumar, "Subsethood-product fuzzy neural inference system (SuPFuNIS)", IEEE Trans. Neural Networks, vol. 13, no. 3, pp. 578-599, May, 2002.

[9] C. T. Lin and C. S. G. Lee, "Reinforcenent structure/parameter learning for neural-network-based fuzzy logic control systems," IEEE Trans. F1izzy Systems, Vol. 2, No. 1 , Feb. 1994, pp. 46-63. 